\title{
Optimal Resource Allocation of the Press
}

\author{
Xue Yang \\ North China Electric Power University (Baoding), Baoding, 071003, China \\ yxscspx@126.com
}

Keywords: Nonlinear Integer Programming; GM (1, 1) Model; Entropy Weight coefficient Method

\begin{abstract}
The paper studies the optimal resource allocation of the press. Four decisive factors are market share, satisfaction, sales and the number of ISBN assigned. We are made to increase the profits of the press and reduce the gap between expected sales and actual sales at the same time. We set the numbers of ISBN as decision variable, considering the limitation of the 4 factors to the ISBN, we develop the optimal allocation model. Finally, taking into account the human factors, most presses can concentrate more on the most popular books, we improve the model at last. In short, the model can solve the problem of the allocation of the press nicely and be applied into some similar problems effectively.
\end{abstract}

\section{Introduction}

The paper is dealt by 4 parts in order to get the model of nonlinear integer programming. The first part is to decide the decision variable and the objective function; the second part is to develop the constraints between the factors and the decision variable; the third part is to solve the optimal allocation of the press, the process is divided into two steps: primarily, we get the allocation for branches, then we get the final solution to each book; the forth part is to improve allocation model by considering some other factors like human influence and more . Through the 4 parts, we get the optimal solution for the press, which is useful to the publishing industry.

\section{Nonlinear Integer Programming}

For the bureau's application submitted for production planning, human resources situation, and analysis of the market information, Press must set reasonable allocation of ISBN number to each branch , the purpose is to make the press gain the best economic benefits. So here with the goal of maximizing the press's economic benefits, namely: to set the profit as the objective function. Besides, in order to improve the accuracy of the model, we should minimize the gap between the planned sales number and the real sales number. The profit of the press and the accuracy of the model can be expressed as followings, and we select the data of a press, here, we called it A press:

$$
\begin{aligned}
& \text { MaxZ }=\sum_{i=1}^{9} a_{i} b_{i} x_{i} \\
& \text { Minf }=\sqrt{\sum_{i=1}^{9}\left(b_{i} d_{i}-x_{i} b_{i}\right)^{2}}
\end{aligned}
$$

Among the formulation, the $a_{i}$ represents average price for branch $i, b_{i}$ represents the sale numbers for the branches which is predicted in the year of 2006. $d_{i}$ is the number of ISBN branch $i$ had applied for. $x_{i}$ is the number of ISBN of the branch i applied for.

\section{Principle Of The Grey Prediction Mainly For The GM(1,1) Model[1]}

By differentiating the develop trend through the systemic factors, Grey Prediction analyzes the data to find the law when the system changes, then generates data sequence of strong regularity, finally, it establish the corresponding differential equation model to predict the future trend of things. 
What we discuss about is the $\operatorname{GM}(1,1)$ model which is typical. we assume the original sequence is $x^{(0)}=\left(\mathrm{x}^{(0)}(1), \mathrm{x}^{(0)}(2), \ldots \mathrm{x}^{(0)}(\mathrm{n})\right), \mathrm{n}$ represents the number of the data

Accumulate the original data to weaken the volatility and randomness of random sequence, and get the new data sequence:

$$
x^{(1)}=\left(x^{(1)}(1), x^{(1)}(2), \ldots, x^{(1)}(n)\right) x^{(1)}(t)=\sum_{k=1}^{t} x^{(0)}(k)(t=1,2, \ldots, n)
$$

Develop the Linear first-order differential equation for the $x^{(1)}(\mathrm{t})$

$$
d x^{(1)} / d t+a x^{(1)}=u
$$

Here, $a$ is the development coefficient, its feasible set is $(-2,2), u$ represents the grey influence. If we can get the value of $a$ and $u$, we can get $x^{(1)}(\mathrm{t})$, and at last we'll predict the value of $x^{(0)}$.

Get the average value of the $x^{(1)}$ and the constant vector $Y_{n}$. To get the parameter $\hat{a}$ by means of least square method.

$\widehat{a}=(a, u)=\left(B^{T} B\right)^{-1} B^{T} Y_{n}$

Put $\widehat{a}$ to $d x^{(1)} / d t+a x^{(1)}=u$, and solve the equation. And the answer is:

$\hat{x}^{(1)}(\mathrm{t}+1)=\left(\mathrm{x}^{(0)}(1)-u / a\right) \mathrm{e}^{-a t}+u / a$

Get the approximate data sequence by $\widehat{x}^{(0)}(\mathrm{t}+1)=\widehat{x}^{(1)}(\mathrm{t}+1)-\widehat{x}^{(1)}(\mathrm{t})$

To test the Grey Model. The following is the model accuracy test table.

Table 1: accuracy table

\begin{tabular}{cccc}
\hline Rank & Relative(q) & Ratio of the variance(C) & Small error probability(P) \\
\hline I & $<0.01$ & $<0.35$ & $>0.95$ \\
\hline II & $<0.05$ & $<0.50$ & $<0.80$ \\
\hline III & $<0.01$ & $<0.65$ & $<0.70$ \\
\hline IV & $>0.20$ & $>0.80$ & $<0.60$ \\
\hline
\end{tabular}

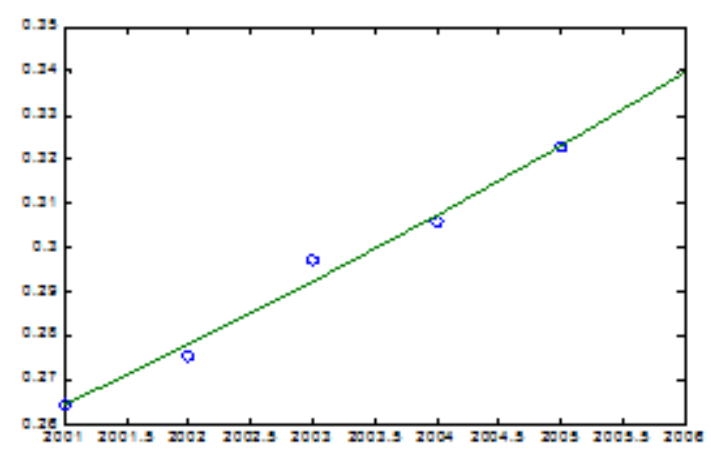

Figure 1: fitted curve from 2001 to 2006 of economy management

By means of GM $(1,1)$ Model, we predict the market share of the branches in the year of 2006 . Figure 1 is the fitted curve.

We can seen from Fig.1 that what we derived from the GM(1,1) Model fit in good condition and meet our requirements

\section{Constraints}

In reality, the numbers of ISBN the press of A had got should be less than what they can get under the stuff's working at $100 \%$ capacity. We can describe this relation that $x_{i} \leq c_{i}, x_{i}$ is the number of ISBN of the branch $\mathrm{i}$ applied, $c_{i}$ is the working capacity of the branch $\mathrm{i}$. 
We conclude that the press must prove half of the numbers of ISBN the branch had applied for. The inequality is $x_{i} \geq d_{i} / 2, d_{i}$ is the number of ISBN branch i had applied.

Apart from the two constraints above, we draw a conclusion that the numbers of ISBN the press of A owed is five hundreds. That is $\sum_{i=1}^{9} x_{i}=500$.

As we all know, the number of ISBN must be integer, thus, let $x_{i}$ be a sequence, where $i=1,2, \cdots, 9$, and $x_{i}$ cannot be decimal.

In general, the branches can subjectively exaggerated the number of ISBN they need, for they can get more benefits, thus it may cause big differences between the number of ISBN planned and allocated actually. Therefore, we give one constraint: $x_{i} \leq d_{i}$.

Considering to maximum the benefits of the press A, we think that the sales number got from the ISBN must be more than the sales number got from the market analysis. So we get the formula: $x_{i} b_{i} \geq\left(\sum_{j=1}^{9} b_{j} x_{j}\right) \cdot e_{i} \cdot g_{i}$

\section{Solutions[2,3]}

All above, objective function is

$$
\left\{\begin{array}{c}
\text { MaxZ }=\sum_{i=1}^{9} a_{i} b_{i} x_{i} \\
\operatorname{Minf}=\sqrt{\sum_{i=1}^{9}\left(b_{i} d_{i}-x_{i} b_{i}\right)^{2}}
\end{array}\right.
$$

The constraints are

$$
\left\{\begin{array}{c}
x_{i} \leq c_{i}, x_{i} \geq d_{i} / 2, x_{i} \leq d_{i}, \sum_{i=1}^{9} x_{i}=500 \\
x_{i} b_{i} \geq\left(\sum_{j=1}^{9} b_{j} x_{j}\right) \times e_{i} g_{i}, x_{i}(i=1,2, \ldots, 9)
\end{array}\right.
$$

Here, $x_{i}$ must be the integer and larger than zero. We use Lingo to solve this nonlinear integer programming ${ }^{[2]}$, then we get the following result.

Table 2: number of the ISBN allocated for the branches

\begin{tabular}{|c|c|c|c|c|c|c|c|c|c|}
\hline Branches & A & B & C & D & E & F & G & H & I \\
\hline Number & 55 & 66 & 120 & 59 & 72 & 43 & 15 & 32 & 30 \\
\hline
\end{tabular}

In the Table 2, a represents Computer, B represents Economic \& Management, C represents Math, D represents English, E represents Political class, F represents Mechanical Energy, G represents Chemical Class, H represents Geography, I represents Environment. Based on the allocation plan above, we get the optimal allocation of each books in the benchmark of the customer satisfaction. Here is the result of the specific distribution.

Table 3: number of the ISBN allocated for all the books of press A

\begin{tabular}{|l|l|l|l|l|l|l|l|l|l|l|l|l|l|l|l|l|l|l|l|l|}
\hline NC & 1 & 2 & 3 & 4 & 5 & 6 & 7 & 8 & 9 & 10 & 11 & 12 & 13 & 14 & 15 & 16 & 17 & 18 & 19 & 20 \\
\hline NI & 8 & 10 & 1 & 1 & 1 & 7 & 11 & 1 & 11 & 4 & 10 & 8 & 2 & 3 & 2 & 3 & 5 & 6 & 22 & 5 \\
\hline NC & 21 & 22 & 23 & 24 & 25 & 26 & 27 & 28 & 29 & 30 & 31 & 32 & 33 & 34 & 35 & 36 & 37 & 38 & 39 & 40 \\
\hline NI & 2 & 6 & 79 & 2 & 7 & 13 & 1 & 1 & 2 & 7 & 33 & 2 & 1 & 7 & 3 & 5 & 1 & 3 & 3 & 1 \\
\hline NC & 41 & 42 & 43 & 44 & 45 & 46 & 47 & 48 & 49 & 50 & 51 & 52 & 53 & 54 & 55 & 56 & 57 & 58 & 59 & 60 \\
\hline NI & 3 & 14 & 7 & 11 & 15 & 12 & 8 & 4 & 10 & 1 & 6 & 14 & 5 & 7 & 1 & 2 & 3 & 1 & 3 & 5 \\
\hline NC & 61 & 62 & 63 & 64 & 65 & 66 & 67 & 68 & 69 & 70 & 71 & 72 & & & & & & & & \\
\hline NI & 3 & 6 & 5 & 7 & 1 & 8 & 7 & 8 & 10 & 4 & 3 & 8 & & & & & & & & \\
\hline
\end{tabular}


In the Table 3, NC represents course number, NI represents number of the ISBN. The meaning of the Course number is as follows:

The course number from 1 to 10 represents: Program design for $\mathrm{C}++$, Program design for $\mathrm{C}$, technology and Application of DSP, Java, Compiler theory, Data Construction, Software Engineering, Single Chip Microcomputer, Multi-media, Artificial intelligence;

The course number from 11 to 20 represents: Insurance, Organizational behavior, The Stock Investment, Western Economics, Enterprise Management, Econometrics, Technological Economics, Financial Management, Economy Management System, International Economics

The course number from 21 to 30 represents: Discrete Mathematics, Mathematical analysis, Higher Mathematics, The ODE, Complex Variable, the Theory of the Probability and Statistics, Abstract Algebra, Economic Mathematics, the Calculus, Linear Algebra;

The course number from 31 to 40 represents: College English, The French, The Translation, Extensive Reading, Computer English, The Oral English, American Literature, The Japanese, Business English, and The Grammar;

The course number from 41 to 50 represents: Modern International Economy and Politics, Deng Xiaoping Theories and the Important Thought of the "Three Represents", Marxism Political Economy Principe, Marxism Philosophic Theory, The Generality of Mao Zedong thoughts, Ideological and Moral Cultivation, Legal Foundation, Political economy, Engineering Drawing, Process Control;

The course number from 51 to 60 represents: Descriptive Geometry, Machine Design, Mechanical Principle, Mechanical Drawing, Chemistry and Modern Civilization, Organic Chemistry, Physical Chemistry, chemical Engineering, Engineering Chemistry, General Chemistry;

The course number 71 and 72 represents: Environmental Chemistry, Environmental management.

\section{Conclusion}

Through the optimal allocation of press A, we deal well with the problem and get the reasonable allocation results, we can see from the Table 2 and 3 that the allocation is targeted, we give weight objectively and use the Nonlinear Integer Programming to work out the result. Thus, the solution of the optimal resource allocation is worth consulting for the publishing industry.

\section{Reference}

[1] Rongbo Tan, Xiaoren Mei. The SPSS Course of the Statistical Analysis. Beijing, China: The Science Press. 2007.

[2] Xingjin Xie. Optimization Model and LINDO /LINGO Software. Beijing, China: Tsinghua University press.

[3] Rongbo Tan, Xiaoren Mei. Guide for SPSS Statistic Analysis. Beijing, China: The Science Press. 2007. 